\title{
Taxonomy of Usability Requirements for Home Telehealth Systems
}

\author{
Jaspaljeet Singh \\ Department of Computer Science \\ University of Auckland \\ Private Bag 92019, Auckland, \\ New Zealand \\ jran055@aucklanduni.ac.nz
}

\author{
Christof Lutteroth \\ Department of Computer Science \\ University of Auckland \\ Private Bag 92019, Auckland, \\ New Zealand \\ lutteroth@cs.auckland.ac.nz
}

\author{
Burkhard C. Wünsche \\ Department of Computer Science \\ University of Auckland \\ Private Bag 92019, Auckland, \\ New Zealand \\ burkhard@cs.auckland.ac.nz
}

\begin{abstract}
Over the past decade increasing healthcare and elderly care costs and demographic changes are rapidly making traditional healthcare concepts unaffordable for many developed countries. Telecare and telehealth applications have become increasingly popular because of their promise to reduce costs and staffing requirements while maintaining or even improving care. Most of the existing applications are centred around the clinical users. Patient factors are considered, but usually only incorporated into the user interface design rather than the system and its application. The patient population, and especially the large proportion of elderly patients, has unique needs, capabilities and limitations that must be considered throughout the design process of such applications. In this paper we present a taxonomy of usability requirements and design concepts for home telehealth systems which enable a more patient centric design. We systematically explore the usability problems of past and current telehealth applications. Problems faced by users of home telehealth systems are identified and solutions delineated. The paper aims to build a good understanding of the technology needs of the elderly population. It represent a solid foundation for constructing novel and more general telehealth solutions in order to make the technology more effective and more widely available.
\end{abstract}

\section{Categories and Subject Descriptors}

H.1.2 [User/Machine Systems]: Human factors;

H.5.2 [User Interfaces]: User-centered design;

J.3 [Life and Medical Sciences]: Health

\section{Keywords}

Telecare, telehealth, usability requirements, patient-centric design

\section{INTRODUCTION}

There is an increasing global demand for more healthcare interventions, especially those that help individuals to improve and take control of their health. Particularly in developed countries, a serious demographic change is taking place, with the elderly population growing significantly. According to the World Health Organization, worldwide the proportion of people aged 60

Permission to make digital or hard copies of all or part of this work for personal or classroom use is granted without fee provided that copies are not made or distributed for profit or commercial advantage and that copies bear this notice and the full citation on the first page. To copy otherwise, or republish, to post on servers or to redistribute to lists, requires prior specific permission and/or a fee.

CHINZ'10, July 8-9, 2010, Auckland, New Zealand.

Copyright 2010 ACM 978-1-4503-0104-6...\$10.00. and over is growing faster than any other age group, and particularly this age group is affected by many chronic diseases [22]. In addition to that, the support ratio for people aged $65+$ is predicted to fall from 9:1 in 2009 and to 4:1 in 2050 [21]. This means that elderly healthcare will pose significant challenges in the future. Telehealth is a promising approach to solve this problem [14, 18].

Unsurprisingly, there has been a significant increase of commercial and research efforts in the field of telehealth. However, much attention is paid to the technical aspects of telecare in the development of new applications, which often leads to less focus on the usability issues. As a result, users have difficulties in adopting potentially useful products. Though much work has been done on meeting the requirements of users, especially elderly ones, there is still a lack of knowledge about their technology needs and how the system should be best designed [9]. In our understanding, usability issues of current telehealth systems have been motivated by medically quantifiable patient parameters, such as vision and motor control. In this paper we take a more holistic view which also incorporates psychological and environmental barriers.

This paper presents a taxonomy of usability requirements for home-based telehealth systems, which will aid development of better telehealth systems in the future. The common barriers that obstruct older patients' abilities to use healthcare systems can be classified as individual limitations, system design limitations, and environmental variables such as social support [13]. The classification helps to form a better understanding of what users expect from healthcare interventions, therefore the common barriers serve as the top level of our taxonomy. We have approached home telehealth systems development through the eyes of users, rather than through the eyes of system designers or health professionals. These requirements are derived from the identification and categorization of common problems faced by older adults when dealing with home telehealth technologies.

Section 2, 3 and 4 cover the three classes of barriers: individual limitations, system limitations and environmental variables respectively. We review the current state-of-the-art, discuss fundamental problems, and suggest solutions. Section 5 concludes the paper.

\section{INDIVIDUAL LIMITATIONS}

The individual limitations in this context refers to any health related problems or other self-inabilities that obstruct users from using the health application effectively. The following are recommendations to overcome this barrier. 


\subsection{Functionality}

\subsubsection{Natural Input}

The telehealth systems should be designed to enable users to interact with the system naturally. Elderly people with tremors or Parkinson's disease experience difficulties in moving the mouse [18]. Kaufman et al. [13] claim that using the keyboard and mouse is a formidable obstacle to some users. One possible solution is to provide touch screen input as is the case for ATI's patient station [2]. With the new Windows 7 operating system such solutions will also become more accessible to standard PC users [7].

\subsubsection{Multilanguage Support}

Language should not be a barrier for users to use telehealth systems [16]. Most of the existing telehealth systems are centred around the Western society, and since the system is presented in English. Although English is a universal language, we need to recognise that there are many health consumers who do not converse in or understand English. It is interesting to note that Tunstall's telehealth monitors support a wide range of different languages and local dialects where needed [20].

\subsubsection{Reminders}

Reminders are a useful feature of telehealth systems. Mynatt et al. [15] claim that elderly face difficulties in remembering tasks such as taking their medication. Current telecare systems usually provide reminders for monitoring sessions and often can be configured to provide medication reminders. A more comprehensive scheduling system could also be envisioned, e.g. to provide reminders to attend to a doctor appointment.

\subsubsection{Reduced Computer Anxiety}

Some older adults have fear and avoid to be confronted with modern technology such as PC-based health application system. Some of the participants of a study on PC-based telehealth system expressed that it would be too difficult for elderly to learn how to use the system [18]. Although the patients were informed that they did not need any computer knowledge to use the system and the system is not a PC, they perceived the system as a computer and expressed their computer anxiety. Many existing telecare systems address this problem by using interfaces which consists of only a screen and a few buttons and hence are more similar to a large digital clock than a computer.

\subsubsection{Customised to Self-efficacy}

Users have different self-efficacies or abilities to use computer systems efficiently. Some users may be slow while others are fast. The system should be customized according to individuals' levels of the system self-efficacy and anxiety [18]. We could meet this requirement by creating a more personalised system by allowing the users to make adjustments to suite their limitations or preference. For instance, there should be more detailed instructions on each stage of the system to cater for users without computer experience. This group of users may need further explanation on terms like "double-click" or "pointer" than users with computer experience. Experienced users should be able to turn off this feature easily.

\subsection{Understandability}

\subsubsection{Mental Model}

The users need to understand how the system works at the outset [13]. This will aid users especially elderly users in getting a rough idea about what is expected from them when using the system.
We could guide the users by having a short video describing how the system works. Otherwise, we could have a separate screen that clearly illustrates and explains how the system works. This piece of information should be part of the system and not a separate user manual sheet.

\subsubsection{Guided Instructions}

Telehealth system should provide sufficient instructions to use the system comfortably. If designed properly then even elderly patients with limited computer experience can use such a system successfully [8]. A helpful feature is audio instructions on each stage of the application [18], which is essential for visual impaired patients. Note that visual impairment is a common side effect of several chronic diseases such as diabetes and occurrences increase with age.

\subsection{Interface Design}

\subsubsection{Readability}

Older adults are more affected or confused by distracting context such as clutter on a screen [13]. This may limit their ability to selectively attend to relevant screen features and perform concurrent tasks such as to hold a conversation with their doctors at the same time. Small font size and links that are inadequately spaced are some known concerns. There should be more white space on the screen to allow users to see important information clearly. The system interface should be designed with high contrasting colors to differentiate the fonts and background and the font size should be big enough to accommodate users with vision problems [16].

\subsubsection{More Pictures than Words}

Important information should be presented with more pictures than words. Avoid using text alone on buttons. Use icons with both graphics and text to aid users to quickly understand what they need to do next. Ojel et al. [17] tested and confirmed that elderly users make fewer errors in using home telehealth care devices when they deal with icons instead of words in the device interface. Thus, telehealth system interface should be designed more graphical than textual.

\subsubsection{Appropriate Use of Colours}

The choice of colours used should not distract users' interaction with the system. Many users, especially elderly ones, have poor colour and contrast sensitivity. Hence, users might not be able to differentiate between different colours, particularly when they are presented with only dark or bright colours. Designers should lighten the light colours and darken the dark colours to increase its visual accessibility [3]. The colours must also have a clear meaning. The interface must take into account cultural aspects, i.e. different interpretations of colours and symbols. For example, red indicates danger in Western society but it means happiness or luck to the Chinese community.

\subsubsection{Clear Screen Transitions}

There should be clear transition between screens or sections of the applications [13]. This will help users to keep track of the flow of the system which otherwise will make them feel wonder or confused about where they are in the system.

\subsection{Operational Support Services}

\subsubsection{Tailored Training Program}

Most of the home telehealth applications incorporate training programs for its users. Older adults may take more time in 
absorbing the training lesson or learning how to use the system. A common recommendation is to conduct a separate training session specifically designed for the older adults. Tailored training components should aim to reduce users' anxiety and improve users' self-efficacy in using the system [18].

\subsubsection{Adequate Technical Support}

The system developers should ensure that there is sufficient technical support available to the users. Elderly patients do not want to be involved with technical facets of the system [1]. Therefore, the technical support staff need to make home visits to solve the technical problems instead of providing detailed step-bystep instructions to the users over the phone or online. The technical support team should also be responsible for the maintenance of the system [18].

\section{SYSTEM LIMITATIONS}

System limitations are characteristics of the technology which prevent the user from making effective use of a system. Below are some of the main recommendations to overcome this problem.

\subsection{Reduced Complexity}

\subsubsection{Easy Data Entry}

Telehealth systems are generally designed to collect health information from the patients. Patients' weight is a common health parameter monitored to avoid numerous health diseases. Some weighing scales use Bluetooth technology to automatically transfer the weight measurements to the device containing the telecare system. It is easier to enter the weight data using this method but the technology is quite expensive. A cheaper solution would be to use a common weigh scale and expect the user to enter the weight measure manually into the system. Simple plausibility tests must be conducted to identify common input errors, e.g. pressing the same key twice (779) or pressing a neighbouring key (69 instead of 79).

\subsubsection{Simplified Tasks}

Users need to feel motivated to use the system. Tedious tasks can cause fatigue and boredom to users [13]. So, every task required to be performed by the user should be simplified. For example, it should be easy for the users to create their health profile with just a few button clicks and as few instructions as possible.

\subsection{Feedback}

\subsubsection{Feedback on Health Status}

Most of the current telehealth systems provide detailed health information to the doctors, but little feedback is given to the patients. Patients are mainly concerned over their health status in using telehealth systems [18]. Hence, upon obtaining the necessary information from a user, the system should show the health status of the user at an appropriate level (amount of details and medical terminology).

\subsubsection{Graphical Representation of Health Status}

A day-to-day graphical representation of users' health data will enable both the clinician and the patient to visualize the health progress better [17]. This could be a powerful tool for communication between the patient and the clinician. It also gives the patient a sense of empowerment which can motivate them to maintain and improve their health. Carefusion's TeleAM system provides graphical representation for health parameter such as blood glucose, blood pressure, and peak flow [6].

\subsection{Non-functional Requirements}

\subsubsection{Reliable Health Measurements}

The system must be stable, interoperate without interruption and provide reliable monitored values [5]. Several participants of a home telecare system study were interested in knowing to what extend the system is reliable in terms of obtaining accurate measures (such as blood pressure) and what processes had been implemented to recognise wrong measurements [18]. So, if the system enables users to measure and record their blood pressure, the system should capture the accurate count.

\subsubsection{Security and Confidentiality}

Telehealth systems should support computer security and data confidentiality [5]. The majority of participants of a computerbased telehealth study agreed that they were not concerned about confidentiality related to the system provided that access to the health information would be limited to relevant medical doctors [18]. Nearly half of the participants of another study on telehealth systems avoided talking about personal issues over the equipment that they would otherwise feel comfortable discussing with a nurse in person [1]. These participants were under the impression that tele-encounters pose similar confidentiality breaches associated with computer-based Internet or electronic mail communications. Therefore, the application should address this requirement and provide some form of evidence to gain the confidence of users.

\section{ENVIRONMENTAL VARIABLES}

Environmental variables are other external challenges that prohibit users from using the system as expected. The following points provide some ways to address this problem.

\subsection{Active User Participation}

There is wide agreement that engaging patients to be an active part of the care process is an essential element of the quality of care [10]. Most current telehealth solutions are designed for the clinicians, in which the patients play a passive role, as providers of their health data instead of actively participating in managing their own health. Patients can be encouraged to take charge of their health by enabling them to ask questions, choose between multiple options, and set goals and take actions to improve their health status. Other examples are diet and exercise plans the user creates according to their own preferences in cooperation with medical experts [19].

\subsection{Multi-User Support}

Many telecare systems are designed for use by one patient only. This increases security and simplifies data input, e.g. if a patient stands on a scale with Bluetooth connectivity the system automatically assumes that the measurement is by the user. However, the costs are multiplied if several household members require telecare. Furthermore, even if only one user requires usage of the system, patient motivation can be increased if several household members participate, since this adds a social dimension to the system. Moreover, a single user system has to be transported when travelling. A multi-user system can be installed at community meeting places which not only increases accessibility but also further increases motivation and social support. We therefore strongly recommend multi-user support [18], e.g. by having individual user logins or swipe card access. 


\subsection{Face-to-face Communication}

There is a strong concern over face-to-face communication between the doctor and the patient [1]. Older adults still want to see and communicate with their health providers when using teleheath. This requirement can be accommodated by incorporating video consultations. Although most of the participants of Agrell et al.'s home telecare study [1] have perceived major differences between "virtual" and in person home nursing visits, they have placed a high value on the video presence of the nurse in home telecare. Intel's Health Guide PHS6000 includes an integrated camera, allowing patients to conduct a video call with their healthcare professionals [12].

\subsection{Social Support}

Older people may receive less human contact when monitoring technology replaces nurse visits. Some users especially those living alone expect telehealth systems to provide some form of social support besides the home-based healthcare [18]. It is evident that the quality of life of elderly benefits from a rich set of social relationships among a variety of individuals [11]. Therefore, existing telecare architectures should be extended to provide awareness and connectedness between older people, their informal carers and family members [4].

\section{RESULTS AND CONCLUSIONS}

Telehealth solutions are increasingly being developed to improve healthcare and overcome its mounting costs. The growing deployment of telehealth interventions helps us to understand specific requirements of health consumers towards these systems. In this research we evaluated existing telehealth systems, identified shortcomings, and created a taxonomy of usability requirements. Problems faced by users were systematically categorised into individual limitations, system limitations and environmental variables. Solution to each set of problems were suggested. A more detailed discussion of the presented issues is found in [23]. The results enable the reader to improve existing systems, to use them more effectively, and to develop novel more effective and efficient telehealth systems.

\section{REFERENCES}

[1] H. Agrell, S. Dahlberg, and A. F. Jerant. Patients' perceptions regarding home telecare. Telemedicine journal and e-health, 6(4):409 - 415, 2000.

[2] AmericanTeleCare. Ati homepage, 2010. http://www.americantelecare.com/

[3] A. Arditi. Effective color contrast: designing for people with partial sight and color deficiencies. Lighthouse international homepage, 2010. http://www.lighthouse.org/

[4] H. Baharin, R. Mühlberger, and A. Loch. Mutuality: A key gap in the move to telecare. Auckland, New Zealand, 2009. ACM SIGCHI New Zealand Chapter's International Conference on Computer-Human Interaction.

[5] T. Botsis, G. Demiris, S. Pedersen, and G. Hartvigsen. Home telecare technologies for the elderly. Journal of Telemedicine and Telecare, 14(7):333 - 337, 2008.

[6] Carefusion. Carefusion homepage, 2010. http://www.carefusion.com/
[7] First looks from the labs: Touch-screen features in windows 7. 2009. http://cnettv.cnet.com/touch-screen-featureswindows-7/9742-1_53-50074521.html

[8] L. S. Evangelista, A. Strömberg, C. Westlake, A. TerGalstanyan, N. Anderson, and K. Dracup. Developing a webbased education and counseling program for heart failure patients. Progress in Cardiovascular Nursing, 21(4):196 201, 2006.

[9] S. K. M. Hägglund. Health informatics and the delivery of care to older people. Maturitas, 63(3):196 - 199, 2009.

[10] J. H. Hibbard and P. J. Cunningham. How engaged are consumers in their health and health care, and why does it matter? Technical report, Center for Studying Health System Change, 2008.

[11] T. Hirsch, J. Forlizzi, E. Hyder, J. Goetz, J. Stroback, and C. Kurtz. Elder project: Social, emotional, and environmental factors in the design of eldercare technologies. Pages 72 - 79. Conference on Universal Usability, 2000.

[12] Intel. The intel health guide. 2010. http://www.intel.com/corporate/healthcare/emea/eng/healthg uide/index.htm

[13] D. R. Kaufman, V. L. Patel, C. Hilliman, P. C. Morin, J. Pevzner, R. S. Weinstock, R. Goland, S. Shea, and J. Starren. Usability in the real world: assessing medical information technologies in patients' homes. Journal of Biomedical Informatics, 36(1 - 2):45 - 60, 2003.

[14] M. Loane and R. Wootton. A review of telehealth. Medical Principles and Practice, 10:163 - 170, 2001.

[15] E. D. Mynatt, I. Essa, and W. Rogers. Increasing the opportunities for aging in place. Arlington, Virginia, United States, 2000. ACM Conference on Universal Usability.

[16] E.-S. Nahm, J. Preece, B. Resnick, and M. E. Mills. Usability of health web sites for older adults. CIN: Computers, Informatics, Nursing, 22(6):326 - 334, 2004.

[17] J. M. Ojel-Jaramillo and J. J. Cañas. Enhancing the usability of telecare devices. Human Technology, 2:103 - 118, 2006.

[18] M. Rahimpour, N. H. Lovell, B. G. Celler, and J. McCormick. Patients' perceptions of a home telecare system. International Journal of Medical Informatics, 77(7):486 498, 2008.

[19] R. J. Shannon. Motivational interviewing: Enhancing patient motivation for behavior change. In International Handbook of Occupational Therapy Interventions, chapter 57. Springer, 2009.

[20] Tunstall. Telecare and telehealth by the tunstall group, 2010 . http://www.tunstall.co.uk/

[21] United Nations. World population ageing 2009.Technical report, New York, 2009.

[22] WHO. Active aging - a policy framework. Technical report, World Health Organisation, Geneva, 2002.

[23] J. Singh, C. Lutteroth, and B. C. Wünsche, A Taxonomy of Usability Requirements for Home Telehealth Systems. Technical Report UoA-SE-2010-1, Software Engineering, University of Auckland, May 2010. 